\title{
Granzyme K: a novel mediator in acute airway inflammation
}

\author{
K Bratke, ${ }^{1}$ A Klug, ${ }^{1}$ P Julius, ${ }^{1}$ M Kuepper, ${ }^{1}$ M Lommatzsch, ${ }^{1}$ G Sparmann, ${ }^{2}$ \\ W Luttmann, ${ }^{1}$ J C Virchow ${ }^{1}$
}

- Supplementary Material and Methods and Results data are published online only at http:// thorax.bmj.com/content/vol63/ issue 11

${ }^{1}$ University Medical Clinics Rostock, Department of Pneumology, Rostock, Germany; ${ }^{2}$ University Medical Clinics Rostock, Department of Gastroenterology, Rostock, Germany

Correspondence to:

Dr K Bratke, Abteilung für Pneumologie, Klinik und Poliklinik für Innere Medizin, Universität Rostock, Ernst-Heydemann-Str 6, 18057 Rostock, Germany; kabratke@med.uni-rostock.de

Received 26 September 2007 Accepted 15 May 2008

Published Online First

17 June 2008

\section{ABSTRACT}

Background: Granzymes are a subfamily of serine proteases involved in the pathogenesis of many inflammatory disorders. In contrast with granzyme $A$ and $B$, the role of granzyme $K(G r K)$ in human lung diseases is unknown. Therefore, the release and expression of GrK in allergic asthma, chronic obstructive pulmonary disease (COPD) and bronchopneumonia were investigated.

Methods: Soluble Grk was quantified using an enzyme linked immunosorbent assay in the bronchoalveolar lavage fluid of patients with allergic asthma (before and after segmental allergen challenge), and in patients with mild COPD, pneumonia and in healthy controls. The molecular form of GrK was analysed by western blot. Flow cytometry was performed to determine the cellular expression of GrK.

Results: Compared with healthy controls, there were normal levels of soluble GrK in the bronchoalveolar lavage fluid of patients with COPD, and patients with allergic asthma before allergen challenge. In contrast, soluble GrK was strongly increased in the bronchoalveolar lavage fluid of patients with acute bronchopneumonia. In patients with allergic asthma, there was a significant increase in soluble GrK as well as in GrK expressing CD8 ${ }^{+} \mathrm{T}$ cells in the bronchoalveolar lavage fluid $24 \mathrm{~h}$ and $72 \mathrm{~h}$ after allergen challenge. After allergen challenge, soluble GrK correlated with the percentage of GrK expressing $\mathrm{CD}^{+} \mathrm{T}$ cells. Finally, it was shown that the endobronchial release of the CCR5 ligand CCL3 might be a mechanism for the recruitment of $\mathrm{GrK}^{+} \mathrm{CD} 8^{+} \mathrm{T}$ cells after allergen challenge. Conclusion: These data provide the first evidence that expression of GrK is upregulated in acute airway inflammation, both in infectious and non-infectious diseases.

Granzymes (Gr) belong to the family of serine proteases and five of them have already been described in humans, granzymes A, B, H, K and M. They are synthesised by a variety of different cell types (eg, T lymphocytes, natural killer cells, basophils, mast cells) and stored in secretory granules which are located in the effector cell cytoplasm. ${ }^{1}$ Granzymes have been shown to induce apoptosis of virus infected cells by cleaving intracellular substrates. In addition, they can trigger monocyte activation ( $\mathrm{GrA}$ ) and/or extracellular matrix degeneration (GrA and GrB). ${ }^{2}$

$A$ role for granzymes $A$ and $B$ in lung diseases such as hypersensitivity pneumonitis (GrA and $\mathrm{GrB}){ }^{3}$ chronic obstructive pulmonary disease (COPD) (GrA and $\mathrm{GrB})^{45}$ and allergic asthma $(\mathrm{GrB})^{67}$ has been reported. In contrast with granzyme A and B, much less is known about $\mathrm{GrK}$ in human diseases. The plasma concentration is elevated during viral infections such as dengue fever and acute cytomegalovirus infection. ${ }^{8}$ In addition, a functional role for GrK in the development of sepsis was suggested. ${ }^{9}$

GrK, which has trypsin-like activity, ${ }^{10}$ is able to induce apoptosis in target cells by cleaving the nucleosome assembly protein SET. ${ }^{11}$ In addition, it was shown that GrK directly processes the proapoptotic molecule bid (BH3 interacting domain death agonist) leading to mitochondria dependent cell death. ${ }^{12}$ It was first discovered and purified from lymphokine activated killer cells in which it was less abundant than granzymes $\mathrm{A}$ and $\mathrm{B} \cdot{ }^{13}$ In peripheral blood, GrK is mainly expressed by memory $\mathrm{CD}^{+} \mathrm{T}$ cells with a $\mathrm{CD} 27^{+} \mathrm{CD} 28^{+} \mathrm{CCR} 5^{\text {high }}$ phenotype and a small population of $\mathrm{CD}^{+}{ }^{+} \mathrm{T}$ cells. ${ }^{14}$ However, there is currently no information about the endobronchial release and expression of this granzyme in human lung diseases. It was the aim of this study, therefore, to investigate the expression and endobronchial release of $\mathrm{GrK}$ in patients suffering from allergic asthma, COPD, bronchopneumonia and in healthy controls. In addition, the human asthma model of segmental allergen challenge was used to analyse the regulation of GrK in acute allergic airway inflammation.

\section{METHODS \\ Subjects}

Twenty-two non-smoking patients with mild allergic asthma were included in the study using the following criteria: (1) airway hyperresponsiveness, (2) positive allergen skin prick tests, (3) elevated total or specific immunoglobulin (Ig) E concentrations, (4) dual reaction after allergen inhalation (forced expiratory volume in $1 \mathrm{~s}\left(\mathrm{FEV}_{1}\right)$ fall of $>20 \%$ of baseline after $5-10 \mathrm{~min}$ and $>15 \%$ after 4-6 h). Inhaled allergen provocation and calculation of the individual provocation dose were performed as described previously. ${ }^{15}$ Inhaled and segmental allergen challenge were separated by at least 4 weeks. Cromoglycate and corticosteroids were withdrawn at least 7 days before challenge. Inclusion criteria for patients with pneumonia were: (1) onset of dyspnoea and/or cough within the past 7 days prior to bronchoscopy, (2) radiological and clinical signs suggesting pneumonia and (3) enhanced concentrations of $C$ reactive protein in serum $(>5 \mu \mathrm{g} / \mathrm{ml})$. Inclusion criteria for patients with COPD were: (1) smoking history of at least 20 pack-years, (2) dyspnoea on exertion, (3) $\mathrm{FEV}_{1} /$ forced vital capacity ratio $<70 \%$ (GOLD stage I and II) and (4) no use of regular medications. Inclusion criteria for healthy controls were: (1) no smoking history, (2) no history of any 
Table 1 Characteristics of the patients with allergic asthma

\begin{tabular}{|c|c|c|c|c|c|c|c|c|c|}
\hline Sex & $\begin{array}{l}\text { Age } \\
\text { (y) }\end{array}$ & $\begin{array}{l}\text { Duration } \\
\text { of } \\
\text { asthma } \\
\text { (y) }\end{array}$ & $\begin{array}{l}\mathrm{FEV}_{1} \\
\text { (I) }\end{array}$ & $\begin{array}{l}\text { FEV }_{1} \\
\text { (\% pred) }\end{array}$ & $\begin{array}{l}\text { Total IgE } \\
\text { (kU/l) }\end{array}$ & $\begin{array}{l}\text { Specific } \\
\text { IgE (kU/l) }\end{array}$ & Allergen & $\begin{array}{l}\text { Dose } \\
\text { (AU) }\end{array}$ & Medication \\
\hline $\mathrm{F}$ & 24 & 11 & 3.3 & 95 & 226 & 56.6 & D pter & 10 & BA \\
\hline$F$ & 22 & 10 & 4.1 & 105 & 545 & 12.6 & Rye & 76 & $\mathrm{BA}$ \\
\hline M & 34 & 24 & 5.7 & 124 & 127 & 35.2 & Birch & 23 & $\mathrm{BA}, \mathrm{IC}$ \\
\hline$F^{*}$ & 24 & 2 & 4.0 & 105 & 64 & 13.8 & D pter & 2 & $\mathrm{BA}, \mathrm{CR}, \mathrm{OAH}$ \\
\hline $\mathrm{F}$ & 26 & 10 & 3.6 & 101 & 121 & 34.5 & Birch & 28 & $\mathrm{BA}, \mathrm{OAH}$ \\
\hline $\mathrm{F}$ & 25 & 2 & 3.9 & 123 & 20 & 5.3 & Birch & 14 & BA, IC \\
\hline M & 37 & 10 & 4.9 & 104 & 490 & $>100.0$ & Birch & 135 & $\mathrm{OAH}$ \\
\hline M & 22 & 1 & 4.6 & 102 & 695 & $>100.0$ & Rye & 60 & None \\
\hline$F$ & 20 & 14 & 3.9 & 112 & 2047 & 27.5 & Birch & 53 & BA, IC \\
\hline M & 23 & 5 & 3.6 & 77 & 548 & 26.1 & Birch & 76 & $\mathrm{BA}$ \\
\hline $\mathrm{F}$ & 23 & 15 & 3.5 & 92 & 564 & 77.1 & D pter & 25 & BA \\
\hline $\mathrm{F}$ & 25 & 17 & 3.2 & 94 & 96 & 4.0 & Birch & 30 & $B A, I C$ \\
\hline$F^{*}$ & 25 & 3 & 4.0 & 105 & 64 & 16.9 & D pter & 2 & $\mathrm{BA}, \mathrm{CR}$ \\
\hline $\mathrm{F}$ & 24 & 21 & 3.5 & 102 & 155 & 5.2 & Rye & 4 & $\mathrm{BA}, \mathrm{CR}$ \\
\hline M & 20 & 13 & 4.8 & 114 & 141 & 18.7 & Rye & 1150 & $\mathrm{BA}, \mathrm{CR}$ \\
\hline$F$ & 23 & 3 & 3.8 & 99 & 83 & 20.9 & Rye & 1430 & $\mathrm{BA}, \mathrm{IC}$ \\
\hline $\mathrm{F}$ & 28 & 10 & 2.8 & 105 & 22 & 6.9 & Birch & 64 & $\mathrm{BA}, \mathrm{CR}$, IC \\
\hline M & 34 & 10 & 4.6 & 114 & 172 & 30.7 & Rye & 46 & $\mathrm{BA}$ \\
\hline M & 24 & 17 & 4.6 & 94 & 368 & 66.3 & D pter & 49 & $\mathrm{BA}, \mathrm{CR}, \mathrm{OAH}$ \\
\hline M & 24 & 4 & 4.8 & 105 & 104 & 8.3 & D pter & 71 & $\mathrm{BA}$ \\
\hline$F$ & 22 & 2 & 3.3 & 110 & 134 & 39.6 & Rye & 340 & IC \\
\hline M & 24 & 16 & 4.5 & 97 & 1335 & $>100.0$ & Birch & 123 & BA \\
\hline M & 23 & 4 & 4.8 & 107 & 622 & 46.8 & D pter & 2180 & BA, IC \\
\hline
\end{tabular}

Sex, age and duration of asthma of the patients, prebronchodilator $\mathrm{FEV}_{1}$, medication prior to the study (BA, CR, IC, OAH), serum levels of total (normal range $<100 \mathrm{kU} /$ ) and allergen specific (normal range $<0.7 \mathrm{kU} /$ ) IgE and the allergen and dose used for segmental allergen challenge.

*One patient participated once in the $24 \mathrm{~h}$ protocol and once in the $72 \mathrm{~h}$ protocol with 15 months in between.

$\mathrm{AU}$, allergen units; $\mathrm{BA}$, inhaled $\beta_{2}$ agonist; $\mathrm{CR}$, cromoglycate; $\mathrm{D}$ pter, Dermatophagoides pteronyssinus; FEV $\mathrm{F}_{1}$ forced expiratory volume in $1 \mathrm{~s}$; IC, inhaled corticosteroid; IgE, immunoglobulin $\mathrm{E} ; \mathrm{OAH}$, oral antihistamine.

respiratory disease and (3) no use of regular medications. All volunteers gave their written informed consent. The study protocol was approved by the local ethics committee.

\section{Segmental allergen challenge}

Segmental allergen challenge was performed as described previously. ${ }^{16}$ Briefly, $2.5 \mathrm{ml}$ of saline were instilled into the left S8 and S4 or S5 segment, and the left S8 was then lavaged using $100 \mathrm{ml}$ of pre-warmed saline. Subsequently, allergen (diluted in $2.5 \mathrm{ml}$ of saline) was instilled into the right S8 and S4 or S5 segment, and the right S8 was lavaged using $100 \mathrm{ml}$ of prewarmed saline after $10 \mathrm{~min}$. The second bronchoalveolar lavage (BAL) was performed in the left and right S4 or S5 segment, $24 \mathrm{~h}(\mathrm{n}=15)$ or $72 \mathrm{~h}(\mathrm{n}=8)$ after challenge. For BAL in nonasthmatic volunteers and BAL fluid (BALF) processing, see online data supplement.

\section{Analysis of intracellular and soluble granzyme K}

Flow cytometric detection of intracellular antigens was performed as previously described. ${ }^{6}$ The following antibodies were used: anti-CD3 allophycocyanin (clone MEM-57; ImmunoTools, Friesoythe, Germany), anti-CD4 peridinin chlorophyll protein (PerCP) (clone SK3; BD Biosciences, Heidelberg, Germany), antiCD8 PerCP (clone SK1; BD Biosciences) and anti-GrK fluorescein isothiocyanate (clone GM24C3; ImmunoTools). The human GrK ELISA was performed as previously described.

\section{Immunoblotting}

BAL samples and recombinant GrK (Alexis Biochemicals, Lörrach, Germany) were mixed with Tris $\mathrm{HCl}, \mathrm{pH}$ 7.5, containing 1\% sodium dodecylsulfate (SDS), 2 mM EDTA and $10 \%$ glycerol, and heated at $95^{\circ} \mathrm{C}$ for 15 min under non-reducing or reducing (by the addition of $5 \%$ mercaptoethanol) conditions.

Table 2 Characteristics of healthy controls and patients with COPD and pneumonia

\begin{tabular}{llcc}
\hline & Healthy controls & COPD & Pneumonia \\
\hline Age (years) & $43(31-62)$ & $50(44-56)$ & $53(18-75)$ \\
Sex (M/F) & $10 / 8$ & $6 / 0$ & $5 / 2$ \\
BALF recovery (ml) & $56(35-71)$ & $53(35-55)$ & $24(14-38)$ \\
Leucocytes $\left(10^{3} / \mathrm{ml}\right)$ & $58(24-115)$ & $188(71-275)$ & $244(33-1583)$ \\
Macrophages $(\%)$ & $90.7(75.6-96.8)$ & $91.7(82.4-98.8)$ & $48.6(38.2-77.0)$ \\
Lymphocytes $(\%)$ & $6.6(2.4-23.8)$ & $4.4(0.6-12.0)$ & $13.7(4.8-15.8)$ \\
Neutrophils $(\%)$ & $1.0(0.0-4.8)$ & $2.3(0.6-5.2)$ & $34.8(3.0-56.8)$ \\
Eosinophils $(\%)$ & $0.0(0.0-1.2)$ & $0.2(0.0-3.2)$ & $2.4(0.2-5.2)$ \\
\hline
\end{tabular}

Age and gender of the volunteers, as well as the characteristics of the BALF obtained. Except for the gender distribution, all parameters are shown as median (range).

BALF, bronchoalveolar lavage fluid; COPD, chronic obstructive pulmonary disease. 


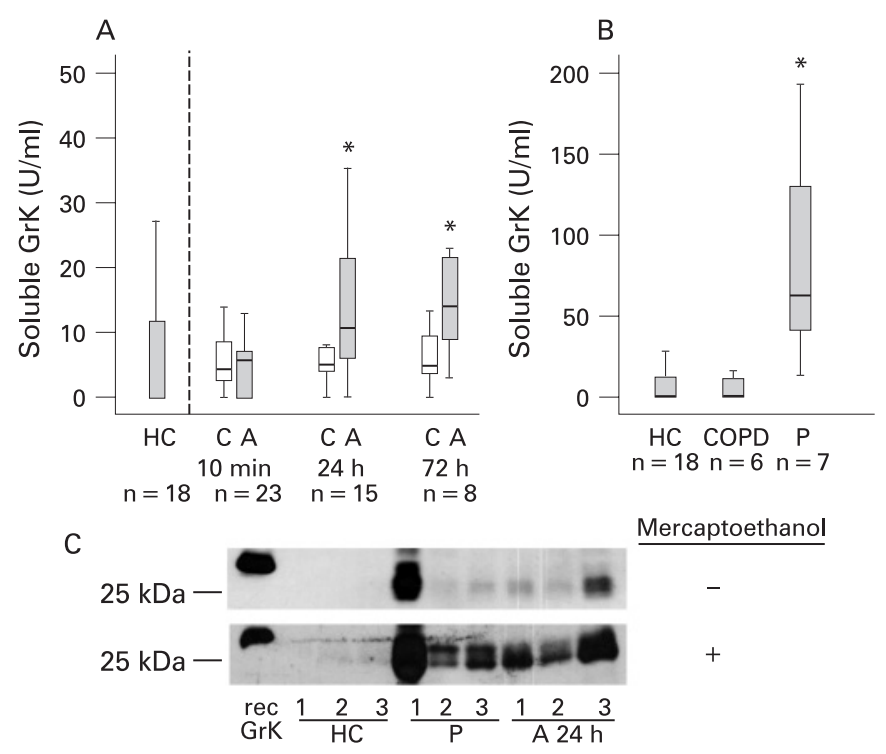

Figure 1 Endobronchial release of granzyme $\mathrm{K}(\mathrm{GrK})$ in human lung diseases. Box plots show the median (line within the box), interquartile range (edges of the box) and range of values less distant than 1.5 interquartile ranges from the upper or lower quartile (vertical lines). Asterisks mark significant differences $(p<0.05)$ between allergen challenged segments and the corresponding control segments as well as healthy controls (A) or between patients and healthy controls (B). (C) Western blot analysis of GrK in bronchoalveolar lavage fluid. $A$, allergen challenged lung segment; $C$, saline challenged lung segment; COPD, chronic obstructive pulmonary disease; $\mathrm{HC}$, healthy control; $\mathrm{P}$, pneumonia. $\mathrm{P}$ values are detailed in the online supplement (table $B$ online).

Proteins were separated by 10\% SDS-polyacrylamide gel electrophoresis and blotted on PVDF membranes (Immobilon-P; Millipore Corp, Bedford, Massachusetts, USA). Blots were incubated with goat anti-granzyme K (C-12; Santa Cruz Biotechnology, Santa Cruz, California, USA) for $2 \mathrm{~h}$ at room temperature. For visualisation of antibody binding, membranes were exposed to horseradish peroxidase labelled anti-goat IgG (GE Healthcare, Munich, Germany) and developed using the enhanced chemiluminescence Plus kit (GE Healthcare).

\section{Statistical analysis}

Data were analysed using SPSS 14.0. Correlation analysis was performed using Spearman's correlation coefficient. Comparison of BALF parameters between allergen and saline challenged control segments was performed using the Wilcoxon signed rank test. For the comparison of soluble GrK levels in BALF of patient groups (asthma, COPD or pneumonia) with healthy controls, the Mann-Whitney U test was performed. Probability values of $p<0.05$ were regarded as significant.

\section{RESULTS}

\section{Characteristics of the participants}

The characteristics of the patients with asthma are shown in table 1 and allergen induced changes in BALF cell counts are detailed in the online supplement (see table A online). Of the six patients with COPD, four patients had GOLD stage I disease $\left(\mathrm{FEV}_{1}\right.$ predicted $>80 \%$ ) and two GOLD stage II $\left(\mathrm{FEV}_{1}\right.$ predicted $<80 \%$ and $>50 \%$ ). In all patients with bronchopneumonia, there was indirect evidence for a bacterial origin of the infection because the patients responded promptly to antibiotic treatment. Microbiological analysis of BALF in this group yielded positive results in three cases (Mycoplasma pneumoniae,
Chlamydia pneumoniae, Legionella pneumophila). Further patient as well as BALF characteristics are detailed in table 2.

\section{Endobronchial concentrations of granzyme $\mathrm{K}$}

Soluble GrK could not be detected in BALF from most healthy controls (median $0.0 \mathrm{U} / \mathrm{ml}$, range 0.0-36.6) (fig 1). In BALF obtained $10 \mathrm{~min}$ after allergen challenge, no differences in GrK concentrations were detected between allergen challenged (A) and saline challenged control (C) segments (C 10 min: median $4.2 \mathrm{U} / \mathrm{ml}$ (range 0.0-13.8); A $10 \mathrm{~min}$ : median $5.4 \mathrm{U} / \mathrm{ml}$ (range $0.0-17.8)$ ). But there was a significant increase in soluble $\mathrm{GrK}$ in BALF $24 \mathrm{~h}$ after allergen challenge (C $24 \mathrm{~h}$ : median $5.0 \mathrm{U} / \mathrm{ml}$ (range 0.0-14.6); A 24 h: median $10.6 \mathrm{U} / \mathrm{ml}$ (range 0.0-129.8)), which persisted $72 \mathrm{~h}$ after allergen challenge (C $72 \mathrm{~h}$ : median $4.8 \mathrm{U} / \mathrm{ml}$ (range 0.0-32.0); A $72 \mathrm{~h}$ : median $13.9 \mathrm{U} / \mathrm{ml}$ (range 3.4-46.2)) (fig 1A). In addition, GrK concentrations in BALF obtained $24 \mathrm{~h}$ and $72 \mathrm{~h}$ after allergen challenge were significantly elevated compared with healthy controls, while no differences were found between baseline GrK levels in patients with asthma and healthy controls (fig 1A).

Furthermore, in BALF from patients suffering from pneumonia, GrK concentrations were significantly upregulated (median $55.2 \mathrm{U} / \mathrm{ml}$ (range 12.6-285.8); $\mathrm{p}=0.001$ ). In contrast, patients with mild COPD did not display elevated GrK concentrations in BALF compared with healthy controls (median $1.2 \mathrm{U} / \mathrm{ml}$ (range 0.0-15.6); $p=1.000$ ) (fig 1B).

Western blot analysis revealed the presence of free GrK in BALF after allergen challenge and in patients with pneumonia but not in healthy controls. Reducing disulfide bonds with mercaptoethanol substantially increased the amount of free GrK, suggesting that GrK is present in BALF in both forms, the free active and the complexed inactive form (fig 1C).

\section{Correlation of soluble granzyme $\mathrm{K}$ with parameters associated with allergic inflammation}

Allergen induced elevated endobronchial GrK concentrations were correlated with the severity of the local inflammatory response, as reflected by a significant correlation between soluble GrK and lymphocyte counts $\left(r_{S}=0.536 ; p=0.039\right)$, eosinophil counts $\left(r_{S}=0.619 ; p=0.014\right)$ and interleukin 5 $\left(r_{S}=0.581 ; p=0.023\right)$ in BALF $24 \mathrm{~h}$ after allergen challenge. No significant correlation between soluble GrK and soluble granzyme $\mathrm{B}$ was found $\left(\mathrm{r}_{S}=0.364 ; \mathrm{p}=0.183\right)$. For concentrations of interleukin 5 and granzyme $B$ and a tabular listing of all $p$ values, see the online supplement (tables $C$ and $D$ online).

\section{Allergen induced increase in BALF GrK ${ }^{+} \mathrm{CD8}^{+} \mathrm{T}$ cells}

Because GrK is mainly expressed by $\mathrm{T}$ cell subsets, we analysed intracellular GrK in $\mathrm{CD}^{+}$and $\mathrm{CD} 8^{+} \mathrm{T}$ cells obtained from BALF $10 \mathrm{~min}, 24 \mathrm{~h}$ and $72 \mathrm{~h}$ after allergen challenge (fig 2). Low percentages of GrK expressing $\mathrm{CD}^{+} \mathrm{T}$ cells were detected in all BAL samples which did not change significantly in response to instilled allergen (fig $2 \mathrm{~B}$ ). Ten minutes after allergen challenge, no differences could be detected in GrK expressing $\mathrm{CD}^{+} \mathrm{T}$ cells from allergen challenged and saline challenged control segments (C 10 min: median $28.3 \%$ (range 17.8-45.4\%); A 10 min: median $25.2 \%$ (range 14.8-43.9\%)). In contrast, $24 \mathrm{~h}$ after allergen challenge, a significant increase in $\mathrm{GrK}^{+} \mathrm{CD} 8^{+} \mathrm{T}$ cells was detected in BAL samples (C $24 \mathrm{~h}$ : median 26.1\% (range 17.4-40.5\%); A $24 \mathrm{~h}$ : median $39.7 \%$ (range 16.8-62.9\%); $\mathrm{p}=0.046)$ which persisted until $72 \mathrm{~h}$ after allergen challenge (C $72 \mathrm{~h}$ : median $32.7 \%$ (range 25.5-51.1\%); A $72 \mathrm{~h}$ : median $47.8 \%$ (range $29.0-76.4 \%$ ); $\mathrm{p}=0.018$ ) (fig $2 \mathrm{~B}$ ). In addition, in 


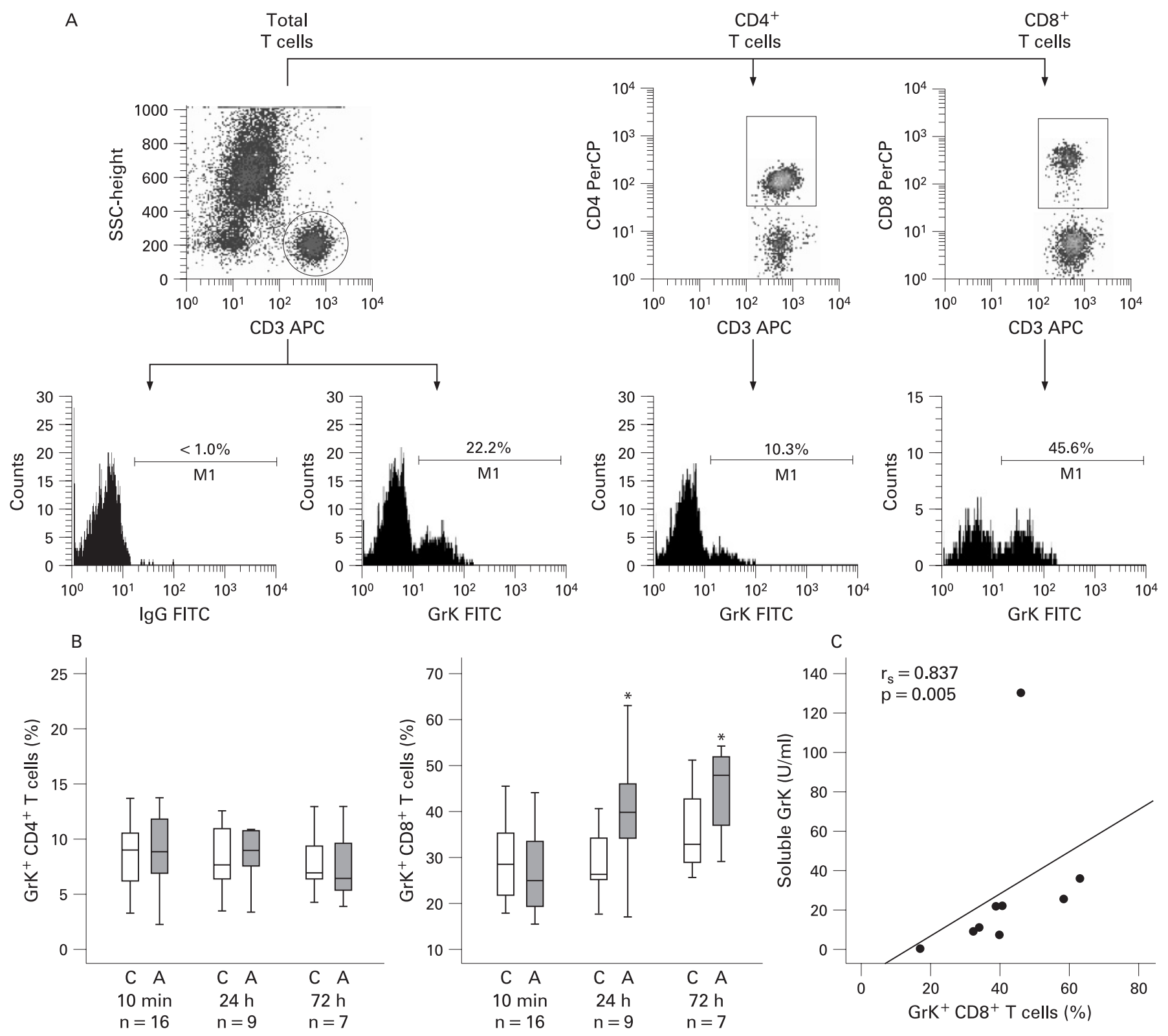

Figure 2 Expression of granzyme $\mathrm{K}(\mathrm{GrK})$ in bronchoalveolar lavage (BAL) derived T cell subsets. (A) T lymphocytes were identified in a SSC/CD3 density plot. Then CD4 and CD8 T cells were further gated and displayed in histograms showing GrK expression in the respective T cell population. (B) Box plots show the median (line within the box), interquartile range (edges of the box) and the range of values less distant than 1.5 interquartile ranges from the upper or lower quartile (vertical lines). Asterisks mark significant differences $(p<0.05)$ between allergen challenged segments and the corresponding control segments. (C) The scatter plot displays the association of soluble GrK with GrK expressing CD8 ${ }^{+} \mathrm{T}$ cells in BAL samples $24 \mathrm{~h}$ after allergen challenge. A, allergen challenged lung segment; C, saline challenged lung segment; FITC, fluorescein isothiocyanate.

BALF, $24 \mathrm{~h}$ after allergen challenge, a positive correlation between soluble GrK and GrK expressing $\mathrm{CD}^{+} \mathrm{T}$ cell subsets was observed (fig 2C). No changes in GrK expression in CD4+ and $\mathrm{CD} 8^{+} \mathrm{T}$ cells from peripheral blood were found $24 \mathrm{~h}$ as well as $72 \mathrm{~h}$ after allergen challenge (data not shown).

\section{Allergen induced release of CCR5 ligands}

Because peripheral blood $\mathrm{GrK}^{+} \mathrm{CD} 8{ }^{+} \mathrm{T}$ cells highly express the chemokine receptor CCR5 (fig 3A) ${ }^{14}$ and preferentially migrate in response to its ligands CCL3 and CCL5, ${ }^{17}{ }^{18}$ we analysed the allergen induced release of CCL3 and CCL5 in BALF. While CCL5 concentrations in BALF were not significantly affected by allergen challenge, a significant increase in CCL3 could be detected $24 \mathrm{~h}$ after allergen challenge (fig $3 \mathrm{~B}$ ). In addition, there was a trend for a positive association between allergen specific CCL3 concentrations (A $24 \mathrm{~h}-\mathrm{C} 24 \mathrm{~h}$ ) and the allergen induced increase in $\mathrm{GrK}^{+} \mathrm{CD}^{+} \mathrm{T}$ cells $\left(\% \mathrm{GrK}^{+} \mathrm{CD}^{+} \mathrm{T}\right.$ cells from $\mathrm{A} 24 \mathrm{~h}$ $\% \mathrm{GrK}^{+} \mathrm{CD} 8^{+} \mathrm{T}$ cells from $\mathrm{C} 24 \mathrm{~h}$ ) which, however, failed to reach statistical significance $\left(r_{S}=0.627 ; p=0.071\right)$.

\section{DISCUSSION}

In the present study we investigated for the first time expression and release of GrK in human lung diseases. Highest GrK concentrations were found in the BALF of patients suffering from bronchopneumonia. Therefore, this study is the first to report elevated concentrations of GrK in infected airways. Together with other studies which investigated changes in GrK plasma concentrations during viral infection 
Figure 3 Allergen induced release of CCR5 ligands. (A) The density plot displays $\mathrm{CD}^{+} \mathrm{CD}^{+}$peripheral blood lymphocytes and shows the coexpression of granzyme K (GrK) and CCR5. (B) Box plots show the median (line within the box), interquartile range (edges of the box) and the range of values less distant than 1.5 interquartile ranges from the upper or lower quartile (vertical lines). Asterisks mark significant differences $(p<0.05)$ between allergen challenged segments and the corresponding control segments. A, allergen challenged lung segment; $C$, saline challenged lung segment; FITC, fluorescein isothiocyanate.
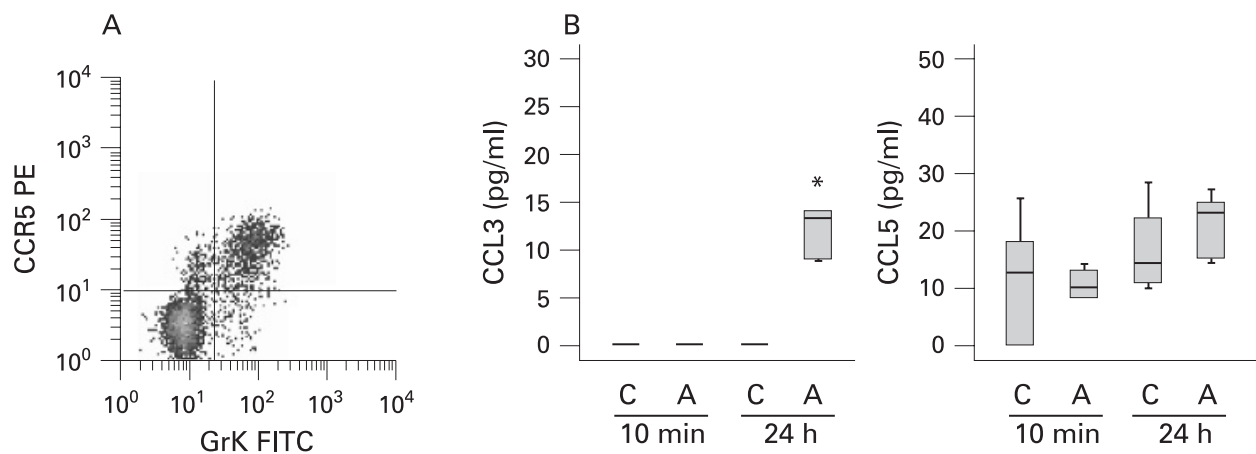

and sepsis, ${ }^{89}$ our data suggest that human GrK is involved in the antibacterial and antiviral host immune response.

In addition, in patients with acute allergic airway inflammation, elevated GrK concentrations were detected which were associated with the severity of the inflammatory response, demonstrating that GrK release is not limited to infections. In contrast, normal GrK levels were found in chronic inflammatory disease patterns as observed in COPD and allergic asthma (without allergen provocation), suggesting that GrK release is a specific event in acute airway inflammation.

Allergen induced GrK concentrations correlate with GrK expressing $\mathrm{CD}^{+} \mathrm{T}$ cells, suggesting that in allergic asthma GrK might originate from these cells. In addition, the low correlation between GrK and GrB concentrations (the latter has previously been reported to be released from basophils in allergic asthma ${ }^{7}$ ) suggests that these two granzymes are indeed not secreted by the same cells. At present it is not clear if the allergen induced increase in $\mathrm{GrK}^{+} \mathrm{CD} 8^{+} \mathrm{T}$ cells in BALF is caused by the proliferation of memory $\mathrm{CD}^{+} \mathrm{T}$ cells in the lung or recruitment of these cells from the periphery. The allergen induced proliferation of BAL T cells in vitro has previously been shown. ${ }^{19}$ In a rat model of asthmatic inflammation, the ovalbumin induced migration of lymphocytes from peripheral blood into the lung has been reported. ${ }^{20}$

In our study, we demonstrated the allergen induced release of the chemokine CCL3, the ligand of the chemokine receptor CCR5, which is highly expressed on $\mathrm{GrK}^{+} \mathrm{CD}^{+} \mathrm{T}$ cells in peripheral blood. ${ }^{14}$ In mouse studies it has previously been reported that CCL3 is important for $\mathrm{CD}^{+} \mathrm{T}$ cell migration to sites of viral infection and into tumours. ${ }^{21}{ }^{22}$ Together, these data suggest that the increase in $\mathrm{GrK}^{+} \mathrm{CD} 8^{+} \mathrm{T}$ cells might be the result of both allergen induced proliferation and recruitment from the periphery through allergen mediated CCL3 release. Notably, we could not find a significant decrease in $\mathrm{GrK}^{+} \mathrm{CD} 8^{+}$ $\mathrm{T}$ cells in peripheral blood $24 \mathrm{~h}$ and $72 \mathrm{~h}$ after allergen challenge, but recruitment from peripheral blood may be an early event which was not detected at later time points.

The free active form of GrK was present in BALF after allergen challenge, but the functional role of extracellular GrK in the pathogenesis of asthma remains unclear. While for granzymes A and B a variety of extracellular substrates have been reported, ${ }^{2}$ no such substrates are known for GrK so far. Because a potent inhibitor of GrK, the inter- $\alpha$-trypsin inhibitor complex, was identified in human plasma, ${ }^{23}$ it can be speculated that extracellular substrates of human GrK might indeed exist. Regarding activity, GrK is highly related to GrA, suggesting that both granzymes may have similar extracellular substrates and function. For extracellular GrA, a role in lymphocyte migration ${ }^{24-27}$ as well as in monocyte, fibroblast and epithelial cell activation has already been reported. ${ }^{28} 29$

In conclusion, we report elevated endobronchial concentrations of human GrK not only in acute bronchopneumonia but also in acute allergic airway inflammation. Allergen induced GrK release was highly associated with GrK expressing CD8 ${ }^{+} \mathrm{T}$ cells in BALF. Finally, the allergen induced release of the CCR5 ligand CCL3 suggests that this mechanism might contribute to the recruitment of $\mathrm{GrK}^{+} \mathrm{CD} 8{ }^{+} \mathrm{T}$ cells to the asthmatic lung.

Acknowledgements: The authors thank P Thamm and E Prestin for excellent technical assistance.

Competing interests: None.

Ethics approval: The study protocol was approved by the local ethics committee.

\section{REFERENCES}

1. Lieberman $\mathbf{J}$. The ABCs of granule-mediated cytotoxicity: new weapons in the arsenal. Nat Rev Immunol 2003;3:361-70.

2. Buzza MS, Bird PI. Extracellular granzymes: current perspectives. Biol Chem 2006;387:827-37

3. Tremblay GM, Wolbink AM, Cormier $Y$, et al. Granzyme activity in the inflamed lung is not controlled by endogenous serine proteinase inhibitors. J Immunol 2000;165:3966-9.

4. Vernooy JH, Möller GM, van Suylen RJ, et al. Increased granzyme A expression in type II pneumocytes of patients with severe chronic obstructive pulmonary disease. Am J Respir Crit Care Med 2007;175:464-72.

5. Hodge S, Hodge G, Nairn J, et al. Increased airway granzyme B and perforin in current and ex-smoking COPD subjects. COPD 2006;4:179-87.

6. Bratke $\mathbf{K}$, Boettcher $\mathrm{B}$, Leeder $\mathrm{K}$, et al. Increase in granzyme $\mathrm{B}^{+}$lymphocytes and soluble granzyme $B$ in bronchoalveolar lavage of allergen challenged patients with atopic asthma. Clin Exp Immunol 2004;136:542-8.

7. Tschopp CM, Spiegl N, Didichenko S, et al. Granzyme B, a novel mediator of allergic inflammation: its induction and release in blood basophils and human asthma. Blood 2006;108:2290-9.

8. Bade B, Lohrmann J, ten Brinke $\mathrm{A}$, et al. Detection of soluble human granzyme $\mathrm{K}$ in vitro and in vivo. Eur J Immunol 2005;35:2940-8.

9. Rucevic M, Fast LD, Jay GD, et al. Altered levels and molecular forms of granzyme $\mathrm{K}$ in plasma from septic patients. Shock 2007;5:488-93.

10. Shi L, Kam CM, Powers JC, et al. Purification of three cytotoxic lymphocyte granule serine proteases that induce apoptosis through distinct substrate and target cell interactions. J Exp Med 1992;176:1521-9.

11. Zhao T, Zhang H, Guo Y, et al. Granzyme K cleaves the nucleosome assembly protein SET to induce single-stranded DNA nicks of target cells. Cell Death Differ 2007;14:489-99.

12. Zhao T, Zhang $\mathrm{H}$, Guo $\mathrm{Y}$, et al. Granzyme $\mathrm{K}$ directly processes bid to release cytochrome $\mathrm{C}$ and endonuclease $\mathrm{G}$ leading to mitochondria-dependent cell death $J$ Biol Chem 2007;282:12104-11.

13. Hameed A, Lowrey DM, Lichtenheld MG, et al. Characterization of three serine esterases isolated from human interleukin 2 activated killer cells. J Immunol 1988;141:3142-7

14. Bratke $\mathbf{K}$, Kuepper $M$, Bade $B$, et al. Differential expression of human granzymes $A$ $\mathrm{B}$, and $\mathrm{K}$ in natural killer cells and during $\mathrm{CD}^{+} \mathrm{T}$ cell differentiation in peripheral blood. Eur J Immunol 2005;35:2608-16.

15. Nassenstein C, Braun A, Erpenbeck VJ, et al. The neurotrophins nerve growth factor, brain-derived neurotrophic factor, neurothrophin-3, and neurotrophin-4 are survival and activation factors for eosinophils in patients with allergic bronchial asthma. J Exp Med 2003;198:455-67. 
16. Lommatzsch $\mathbf{M}$, Julius $P$, Kuepper $M$, et al. The course of allergen-induced leukocyte infiltration in human and experimental asthma. J Allergy Clin Immunol 2006;118:91-7.

17. Taub DD, Conlon $\mathrm{K}$, Lloyd AR, et al. Preferential migration of activated $\mathrm{CD}^{+}$and CD8+ T cells in response to MIP-1 alpha and MIP-1 beta. Science 1993;260:355-8.

18. Fukada K, Sobao Y, Tomiyama H, et al. Functional expression of the chemokine receptor CCR5 on virus epitope-specific memory and effector CD8 ${ }^{+} \mathrm{T}$ cells. J Immunol 2002; 168:2225-32

19. Till SJ, Durham SR, Rajakulasingam K, et al. Allergen-induced proliferation and interleukin- 5 production by bronchoalveolar lavage and blood $T$ cells after segmental allergen challenge. Am J Respir Crit Care Med 1998;158:404-11.

20. Schuster M, Tschernig T, Krug N, et al. Lymphocytes migrate from the blood into the bronchoalveolar lavage and lung parenchyma in the asthma model of the Brown Norway rat. Am J Respir Crit Care Med 2000;161:558-66.

21. Trifilo MJ, Bergmann CC, Kuziel WA, et al. CC chemokine ligand 3 (CCL3) regulates CD8+T-cell effector function and migration following viral infection. J Virol 2003;77:4004-14.

22. Maric M, Chen L, Sherry B, et al. A mechanism for selective recruitment of CD8 T cells into B7-1-transfected plasmacytoma: role of macrophage-inflammatory protein 1alpha. J Immunol 1997;159:360-8.
23. Wilharm E, Marina PAA, Friebel $\mathrm{R}$, et al. Generation of catalytically active granzyme $\mathrm{K}$ from Escherichia coli inclusion bodies and identification of efficient granzyme $\mathrm{K}$ inhibitors in human plasma. J Biol Chem 1999;274:27331-7.

24. Simon MM, Prester M, Nerz G, et al. Release of biologically active active fragments from human plasma-fibronectin by murine T cell-specific proteinase 1 (TSP-1). Biol Chem Hoppe Seyler 1988;368:107-12.

25. Simon MM, Kramer MD, Prester M, et al. Mouse T-cell associated serine proteinase 1 degrades collagen type IV. A structural basis for the migration of lymphocytes through vascular basement membranes. Immunology 1991;73:117-19.

26. Brunner G, Simon MM, Kramer MD. Activation of pro-urokinase by the human T cellassociated serine proteinase HuTSP-1. FEBS Lett 1990;260:141-4.

27. Vettel U, Brunner G, Bar-Shavit R, et al. Charge-dependent binding of granzyme A (MTSP-1) to basement membranes. Eur J Immunol 1993;23:279-82.

28. Sower LE, Froelich CJ, Allegretto N, et al. Extracellular activities of human granzyme A. Monocyte activation by granzyme A versus $\alpha$-thrombin. J Immunol 1996;156:2585-90.

29. Sower LE, Klimpel GR, Hanna WD, et al. Extracellular activities of human granzymes. I. Granzyme A induces IL-6 and IL-8 production in fibroblast and epithelial cell lines. Cell Immunol 1996;171:159-63.

\section{Lung alert}

\section{Mannose-binding lectin deficiency increases mortality risk from pneumococcal infection}

Low serum levels or genotypic variants of the innate immune molecule mannose-binding lectin (MBL) have been associated with increased susceptibility to infectious diseases. Few studies of MBL have included sufficiently large sample sizes and conclusions drawn have been conflicting. This study set out to reanalyse existing data from studies on MBL in order to define the serum level of MBL deficiency and determine the risk of death from sepsis due to this deficiency.

To initially define the serum concentration of MBL deficiency, data on 1642 healthy patients from four out of a possible seven studies was reassessed. An MBL serum concentration of $<0.50 \mu \mathrm{g} / \mathrm{ml}$ was found to be predictive of low producing $M B L 2$ genotypes. Data from six further studies on the association between MBL deficiency and bacterial infection or septicaemia were subsequently reanalysed; 477 heterogeneous patients, with infections ranging from pneumococcal sepsis to undefined septic shock, were included. A serum MBL concentration below this cut-off value within $48 \mathrm{~h}$ of admission was associated with an increased likelihood of death in patients with severe bacterial infections. However, even among survivors, $30 \%$ exhibited serum levels consistent with MBL deficiency. In a separate analysis of patients with severe pneumococcal infection, those with MBL deficiency showed an increased risk of death following adjustment for bacteraemia and co-morbidity.

This study helps delineate a serum level for MBL deficiency and suggests that MBL-deficient patients with severe pneumococcal infection may be an important target for supplementation therapy in the future. However, even this meta-analysis is not without its limitations. The population included is very heterogeneous and different MBL testing methodologies were used in different studies. Nonetheless, as recombinant human MBL replacement therapy is being developed, having an agreed level for replacement and concurrence on what is MBL deficiency is important.

- Eisen DP, Dean MM, Boermeester MA, et al. Low serum mannose-binding lectin level increases the risk of death due to pneumococcal infection. Clin Infect Dis 2008;47:510-6

\section{R M Jones}

Correspondence to: Dr R M Jones, Clinical Research Fellow, Morriston Hospital, Swansea, UK; drmatjones@hotmail.com 